\title{
Right superior vena cava draining into the left atrium in a patient with sinus venosus defect
}

\author{
Meletios Kanakis ${ }^{1}$, Thomas Martens ${ }^{2}$, Cleo Laskari ${ }^{3}$, Theofili Kousi ${ }^{4}$, Afroditi Karafotia ${ }^{4}$, \\ Dimitrios Bobos ${ }^{4}$, and Nicholas Giannopoulos ${ }^{4}$ \\ ${ }^{1}$ Onassis Cardiac Surgery Center \\ ${ }^{2}$ University Hospital Ghent Heart Center \\ ${ }^{3}$ Onassis Cardiac Surgery Center, Athens, Greece \\ ${ }^{4}$ Onassis Cardiac Surgery Centre
}

July 31, 2020

\begin{abstract}
Right superior vena cava draining in the left atrium is a rare anomaly, commonly associated with other cardiac defects. Herein we present the case of a 9 year old patient, asymptomatic but with right heart dilation with sinus venous defect, bilateral superior vena cavas with the right draining into the left atrium.
\end{abstract}

Right superior vena cava draining into the left atrium in a patient with sinus venosus defect

Meletios Kanakis ${ }^{1}$, Thomas Martens ${ }^{2}$, Cleo Laskari ${ }^{3}$, Theofili Kousi ${ }^{4}$, Afroditi Karafotia ${ }^{4}$, Dimitrios Bobos $^{1}$, Nicholas Giannopoulos ${ }^{1}$

1 Department of Pediatric and Congenital Heart Surgery, Onassis Cardiac Surgery Center, Athens, Greece

2 Department of Cardiac Surgery, Ghent University Hospital, Ghent, Belgium

3 Department of Pediatric Cardiology, Onassis Cardiac Surgery Center, Athens, Greece

4 Department of Anesthesiology, Onassis Cardiac Surgery Center, Athens, Greece

Corresponding Author

Thomas Martens

Thom.martens@ugent.be

A 9-year-old asymptomatic boy underwent a screening check-up in order to be eligible for swimming, revealing a holosystolic murmur. Echocardiography demonstrated a sinus venosus defect with moderate dilation of the right cardiac chambers and a left to right shunt. A persistent left superior vena cava was also present draining to the coronary sinus, the right superior vena cava was draining to the left atrium(Figure 1). Surgery confirmed this diagnosis and subsequently a Warden procedure was performed re implanting the right sided superior vena cava to the right atrium. Transesophageal echo confirmed a good surgical result, the postoperative course was uneventful.

Drainage of the systemic venous system into the left atrium (LA) is a rare condition with occasionally a persistent left SVC draining into the LA (1). It is extremely rare for the right SVC to drain into the LA $(2,3)$. The latter is often associated with other cardiac defects. Diagnosis is confirmed with echocardiography and computed tomography or magnetic resonance imaging. 
Clinical presentation varies, depending on the presence of other congenital heart defects. In our case, the presence of bilateral SVCs with a bigger left SVC, together with the sinus venosus and PFO, probably was responsible for absence of clinical symptoms. However, cyanosis is possible, as was seen in a 2-year-old girl with a similar configuration (3), due to a prominent right to left shunt via the drainage of the right SVC to the LA considering that the sinus venosus defect was quite small.

Figure 1

\section{Panel A}

Transthoracic echo showing the sinus venosus defect and the right superior vena cava draining to left atrium (RSVC: right superior vena cava, RA: right atrium, LA: left atrium, ASD: atrial septal defect, sinus venosus defect, CoS: coronary sinus).

\section{Panel B}

Transesophageal echo depicting the reimplanted right superior vena cava draining to the right atrium (SVC: right superior vena cava, RA: right atrium, LA: left atrium)

\section{Panel C}

Intraoperative photo showing the right superior vena cava draining to the left atrium (RSVC: right superior vena cava, LA: left atrium)

\section{Authors' Note}

The parents have consented to publication of the report.

\section{Declaration of Conflicting Interests}

None.

\section{References}

1. Hulten A,Pinto G,Weissman,Fuisz A. Anomalous vena caval return to the left atrium. Circulation 2012; 125: e525-2528.

2. Baggett C,Skeen SJ,Gantt DS,Trotter BR,Birkemeier KL. Isolated right superior vena cava drainage into the left atrium diagnosed noninvasively in the peripartum period. Tex Heart Inst J.2009;36:611-4.

3. Alghamdi MH,Elfaki W,Al-Habshan F,Aljarallah AS. Bilateral superior vena cava with right superior vena cava draining into left atrium. J Saudi Heart Assoc.2015;27:123-6. 


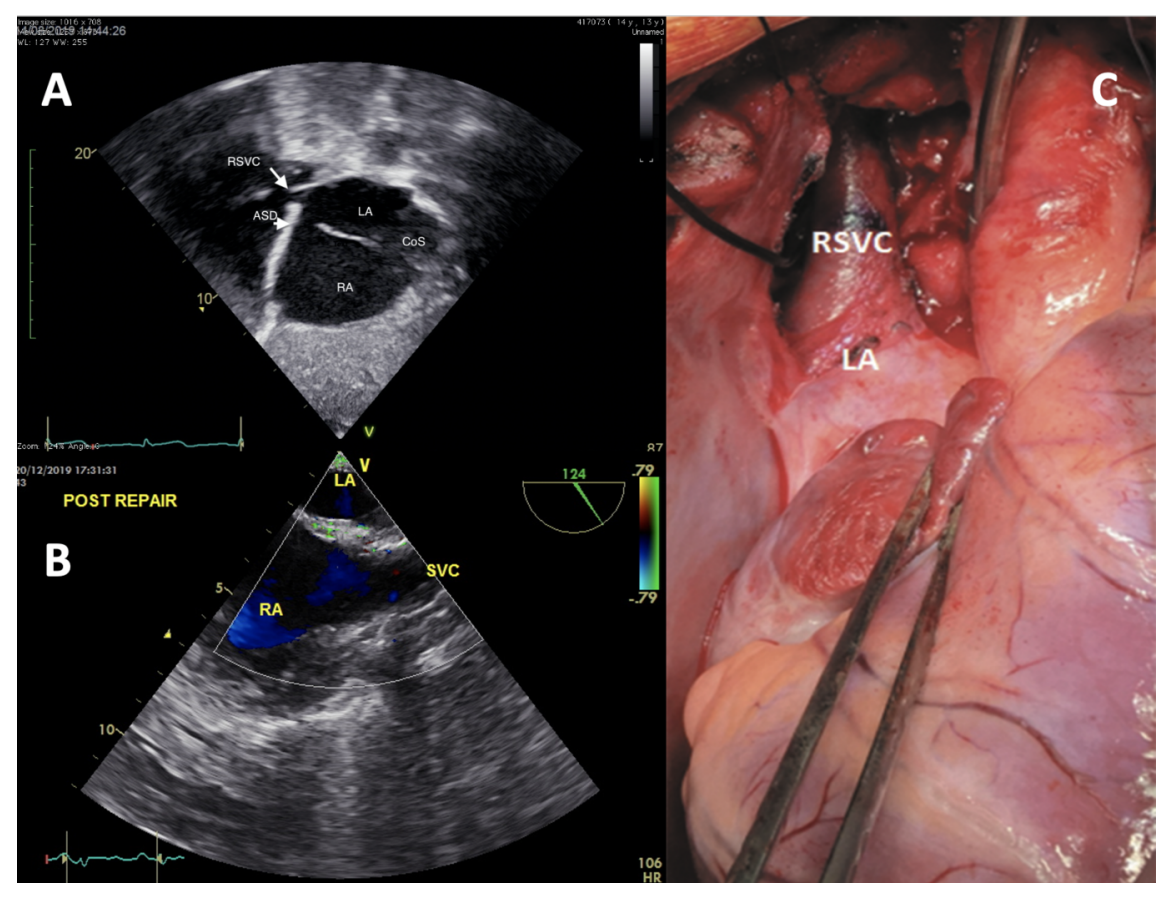

